\title{
actualización
}

\section{Compromiso del sistema nervioso en vasculitis asociadas a ANCA}

[Trabajo monográfico de la carrera de médicos especialistas en Reumatología, UBA-SAR]

\section{María Fernanda Quaglia}

Servicio de Reumatología, Hospital Ramos Mejía, Ciudad de Buenos Aires

Palabras clave:

vasculitis, sistema nervioso, ANCA

Key words:

vasculitis, nervious system, ANCA

\section{R E S U M E N}

Las vasculitis sistémicas son un grupo heterogéneo de enfermedades que se caracterizan por presentar inflamación de los vasos sanguíneos, provocando el daño de la pared vascular. Pueden afectar cualquier tipo de vaso del organismo, por lo cual el compromiso se da en varios órganos o sistemas. Estos procesos presentan una alta mortalidad que se ve reducida con el diagnóstico y tratamiento precoz. Se presenta una revisión con las pautas diagnósticas y alternativas terapéuticas.

\section{A B S T R A C T}

Systemic vasculitis are a heterogeneous group of diseases that are characterized by inflammation of the blood vessels, causing damage to the vascular wall. The causes or the type of vessel of the organism by which the commitment is given in various organs or systems. These processes have a high mortality that is minimized with the diagnosis and early treatment. A review is presented with the diagnostic guidelines and therapeutic alternatives. 


\section{Introducción}

Las vasculitis sistémicas son un grupo heterogéneo de enfermedades que se caracterizan por presentar inflamación de los vasos sanguíneos, provocando el daño de la pared vascular. Pueden afectar cualquier tipo de vaso del organismo por lo cual el compromiso se da en varios órganos o sistemas. Estos procesos presentan una alta mortalidad que se ve reducida con el diagnóstico y tratamiento precoz. Esto podría mejorarse con criterios de clasificación definidos, los cuales están aún en desarrollo.

En ausencia de estos criterios, los criterios propuestos por el ACR y las definiciones o nomenclatura propuestas en el consenso de Chapel Hill se han utilizado como sustitutos. Los avances en el conocimiento de las vasculitis han forzado la realización de un nuevo consenso, que se realizó en 2012, con el fin de mejorar la nomenclatura y cambiar términos y definiciones.

Terminología aceptada para las vasculitis en la Conferencia de Consenso Internacional de Chapel Hill, 2012, sobre la nomenclatura de las vasculitis

Vasculitis de Vaso Grande - Arteritis de Takayasu Arteritis de Células Gigantes

\begin{tabular}{|c|}
\hline $\begin{array}{l}\text { Vasculitis de Vaso Mediano } \\
\text { - Poliarteritis Nodosa } \\
\text { - Enfermedad de Kawasaki }\end{array}$ \\
\hline $\begin{array}{l}\text { Vasculitis de Vaso Pequeño } \\
\text { - Vasculitis Asociadas a ANCA: } \\
\text { Poliangeítis Microscópica } \\
\text { Granulomatosis con Poliangeítis (de Wegener) } \\
\text { Granulomatosis Eosinofílica con Poliangeítis (Churg-Strauss) } \\
\text { - Vasculitis por Inmunocomplejos: } \\
\text { Enfermedad antimembrana basal Glomerular } \\
\text { Vasculitis crioglobulinémica } \\
\text { Vasculitis IgA (Schönlein-Henoch) }\end{array}$ \\
\hline $\begin{array}{l}\text { Vasculitis de Vaso Variable } \\
\text { - Enfermedad de Behcet } \\
\text { - Síndrome de Cogan }\end{array}$ \\
\hline $\begin{array}{l}\text { Vasculitis de Órgano Único } \\
\text { - Angeítis leucocitoclástica cutánea } \\
\text { - Arteritis cutánea } \\
\text { - Vasculitis primaria del sistema nervioso central } \\
\text { - Aortitis aislada }\end{array}$ \\
\hline $\begin{array}{l}\text { Vasculitis asociadas a Enfermedad Sistémica } \\
\text { - Vasculitis lúpica } \\
\text { - Vasculitis reumatoidea } \\
\text { - Vasculitis sarcoidea } \\
\text { - Otras }\end{array}$ \\
\hline $\begin{array}{l}\text { Vasculitis asociadas a Etiología Probable } \\
\text { - Vasculitis crioglobulinémica asociada a VHC } \\
\text { - Vasculitis asociada a VHB } \\
\text { - Aortitis asociada a sífilis } \\
\text { - Vasculitis por inmunocomplejos asociada a fármacos } \\
\text { - Vasculitis asociada a ANCA por fármacos } \\
\text { - Vasculitis asociada a cáncer } \\
\text { - Otras }\end{array}$ \\
\hline
\end{tabular}

Consideraciones adicionales dentro de la clasificación de las vasculitis incluyen datos demográficos de los pacientes, presencia o ausencia de inflamación granulomatosa, la participación, o no, de complejos inmunes y el hallazgo de autoanticuerpos.

Dentro del hallazgo de autoanticuerpos, se encuentran las vasculitis asociadas a ANCA (Anticuerpos contra el citoplasma de los neutrófilos). Estas vasculitis se caracterizan por afectar a vasos de pequeño y mediano calibre.

En el grupo las vasculitis asociadas a ANCA se diferencian tres entidades: la Granulomatosis con Poliangeítis (GPA), la Poliangeítis Microscópica (MPA) y la Granulomatosis Eosinofílica con Poliangeítis (EGPA).

\section{Desarrollo}

\section{Introducción a las vasculitis ANCA}

Las vasculitis asociadas a ANCA es un grupo de condiciones de carácter sistémico que se caracterizan por la inflamación y necrosis de los vasos de mediano y pequeño calibre, y por su asociación con autoanticuerpos dirigidos contra los gránulos citoplasmáticos de los leucocitos polimorfonucleares neutrófilos y los monocitos humanos, como proteinasa 3 (PR3), mieloperoxidasa (MPO), lactoferrina y otras proteasas. La técnica de ELISA permite identificar anticuerpos anti-PR3 y anti-MPO. El $80-90 \%$ de los sueros C-ANCA reconoce la enzima PR3, aunque algunos contienen solo anticuerpos anti-MPO. Entre un $40 \%$ y un $70 \%$ de los sueros P-ANCA contienen anticuerpos anti-MPO. Los ANCA se asocian significativamente con el desarrollo de vasculitis o glomerulonefritis necrosantes, pero se detectan en otras enfermedades. La combinación de los anticuerpos C-ANCA/anti-PR3 y P-ANCA/anti-MPO tiene una sensibilidad del $72 \%$ y una especificidad del $98 \%$ en el diagnóstico de pacientes con sospecha clínica de vasculitis asociada a ANCA o glomerulonefritis pauciinmune. La concentración de anticuerpos anti-PR3 o anti-MPO puede relacionarse con la actividad clínica en algunos pacientes, aunque la utilidad de estos anticuerpos para predecir o prevenir las recidivas aún no ha sido establecida.

Las primeras descripciones de la GPA, MPA y EGPA se dieron en 1930, 1940 y 1950, respectivamente.

Este grupo de entidades se considera la forma más común de vasculitis sistémica primaria de pequeños vasos en adultos. La incidencia anual de las vasculitis asociadas a ANCA, con tendencia en aumento en los últimos años, es de 20 casos por 1.000 .000 de habitantes, siendo de entre 2,1 a 15 en GPA, 2,1 a 17,5 en MPA y 0,5 a 3,1 en EGPA. El pico de incidencia de las mismas se observa en el grupo etario de 55 a 74 años. La mortalidad en el primer año excede el 15\%, el $50-66 \%$ de los pacientes evolucionan a insuficiencia renal crónica terminal y la recurrencia de la enfermedad es del orden del 30 al 50\%.

No existe un patrón evolutivo característico en ninguna de estas enfermedades, las cuales pueden tener un curso clínico rápido, especialmente en el caso de presentaciones neumorrenales, o muy solapado, como en la EGPA, en el cual 
los enfermos pasan por una fase alérgica (asma o rinitis) antes de las manifestaciones generalizadas. La presentación, el número y la extensión de la afección de los órganos son multivariados y, por tanto, el diagnóstico no siempre es fácil; pueden confundirse con un gran número de otros padecimientos autoinmunes, infecciosos o neoplásicos.

\section{Manifestaciones clínicas de las vasculitis ANCA}

Síntomas generales y articulares. No es infrecuente encontrar en casi todos los pacientes síntomas sistémicos como mialgias, artralgias, malestar general, astenia y fiebre, que ocurre hasta en el $50 \%$ de los pacientes. La pérdida de peso es frecuente y se relaciona con las fases activas. El compromiso articular se encuentra hasta en un tercio de los pacientes y es generalmente simétrico y afecta pequeñas articulaciones.

Piel. Las manifestaciones cutáneas en la GPA se presentan en el 10-20\% y hasta en el 50\% de los casos. Las lesiones son variadas y pueden ser vesiculares, papulares, nodulares o ulcerativas. La púrpura es clásicamente palpable. Las lesiones en piel tienden a seguir paralelamente las alteraciones de otros órganos. En la poliangeítis microscópica, la frecuencia de manifestaciones cutáneas es mayor, hasta en $60 \%$ de los pacientes. Las lesiones son similares a las anteriores; también se observa livedo reticularis en $15 \%$ de los casos. En la EGPA se observan erupciones maculopapulares, púrpura y nódulos.

Ojos. La afección ocular es una de las características más típicas de la GPA, aunque es significativa en menos del $20 \%$ de los enfermos. Otras estructuras oculares afectadas son la córnea, la úvea y la esclerótica; en la córnea, la úvea y la esclerótica también pueden estar comprometidas y, con frecuencia, causan síntomas persistentes, refractarios e incapacidades. La retina rara vez se encuentra afectada, lo que puede representar alteraciones coroideas. Por otro lado, en las otras dos enfermedades que nos ocupan, la afección ocular es rara.

Vías aéreas superiores y oídos. Son estructuras característicamente comprometidas en la GPA y EGPA; en la primera existe compromiso en el $75 \%$ de los enfermos al inicio del proceso. En la EGPA, la presencia de rinitis alérgica ocurre en el $70 \%$ de los pacientes, aproximadamente, y puede asociarse con poliposis y obstrucción nasal. El asma es característica fundamental de esta enfermedad y es la manifestación inicial en muchos pacientes; no es infrecuente que permanezca por años, antes de que la enfermedad comprometa otros órganos. No obstante, los cambios destructivos son pocos, a diferencia de lo que ocurre en la GPA, con la sinusitis a repetición, especialmente si se acompaña de dolor en la nariz y cambios erosivos, además de no responder a los tratamientos convencionales. La sinusitis está presente en más del $90 \%$ de los pacientes en la GPA y de no tratarse específicamente puede ocasionar daños irreversibles con importante destrucción de las estructuras óseas. En la MPA menos del 2\% de los enfermos presentan sinusitis como manifestación clínica relevante Las alteraciones del tabique nasal, con colapso del mismo.

La tráquea y los bronquios también se afectan por inflamación importante, pero una vez resueltas, el proceso de cicatrización puede ser tan importante, que ocasionan síntomas por estenosis subglótica o traqueal. Estos cambios se observan hasta en $15 \%$ a $20 \%$ de los pacientes con GPA. Esta complicación puede no correlacionarse con actividad en otros órganos. Se ha encontrado que un alto porcentaje de pacientes con MPA presentan inicialmente afección laríngea (tos, disfonía), en comparación con los pacientes con GPA.

También las estructuras auditivas se encuentran frecuentemente comprometidas en las tres enfermedades. La pérdida súbita de la audición puede ser una manifestación importante.

Pulmón. Cerca de la mitad de los pacientes con GPA pueden presentar alteraciones pulmonares manifestadas por infiltrados intersticiales o nódulos, los cuales, de persistir el proceso inflamatorio, pueden necrosarse y ocasionar cavernas. En los casos más avanzados de daño pulmonar, la hemorragia extensa puede ser una condición irreversible y causa de muerte. Aproximadamente, $10 \%$ a $15 \%$ de los enfermos con GPA O MPA pueden presentar pleuritis con derrame en grado variable. La mayoría de los pacientes con MPA y hemorragia pulmonar tienen también daño renal (causa importante del síndrome neumorrenal). La EGPA tiene una alta frecuencia de manifestaciones pulmonares que puede llegar hasta el $75 \%$ y la pleura se afecta con mayor frecuencia que en las otras dos enfermedades. También puede haber hemorragia pulmonar. Es evidente que la presencia de infiltrados eosinofílicos obliga al diagnóstico diferencial de otras enfermedades infiltrativas por estas células, como el síndrome de Loffler o la neumonía eosinofílica crónica, aunque la aparición de otras manifestaciones pondrá de manifiesto que se trata de una EGPA.

Aparato cardiovascular. Es rara la afección cardíaca en la GPA y la MPA, se menciona insuficiencia cardíaca hasta en el $17 \%$. Rara vez puede haber compromiso de las arterias coronarias o alteraciones en el sistema de conducción. En la EGPA existe una alta frecuencia de alteraciones cardíacas que pueden encontrarse hasta en el $50 \%$ de los casos (pericarditis en $30 \%$ ). La presencia de esta manifestación se debe a miocardiopatía eosinofílica infiltrativa que puede manifestarse como miocarditis o insuficiencia cardíaca secundaria como también en casos de vasculitis coronaria. Recientemente parece reconocerse en el sistema vascular periférico una mayor predisposición a fenómenos trombóticos en pacientes con GPA. Esto podría tener explicación por ciertos fenómenos que favorecen la activación del sistema de coagulación, mediado por los ANCA.

Aparato digestivo. No es frecuente encontrar síntomas digestivos en la GPA, pero cuando ocurren hemorragia alta o baja, dolor abdominal o diarrea, son indistinguibles de los que presentan en la MPA o la EGPA.

Sistema genitourinario. Las manifestaciones renales son de gran importancia en estas enfermedades. El riñón se encuentra comprometido en las etapas iniciales de la enfermedad hasta en una quinta parte de los pacientes con GPA y cerca del $75 \%$ presentan alteraciones en alguna etapa de la enfermedad. En la MPA, el riñón es el órgano que se afecta con mayor frecuencia. En la EGPA, 50\% de los enfermos tienen compromiso renal. 
En cualquiera de las tres enfermedades puede manifestarse en forma abrupta y grave.

Sistema hematológico. La eosinofilia es característica primordial en la EGPA y constituye un criterio en el diagnóstico.

La anemia se observa habitualmente en forma leve; es normocítica, normocrómica y se encuentra en el $20-30 \%$ de los pacientes con GPA y MPA.

En los pacientes con GPA, especialmente, puede haber leucocitosis y trombocitosis, pero las alteraciones hematológicas más frecuentes y graves son producto del tratamiento.

\section{Compromiso del sistema nervioso en las vasculitis ANCA}

En cualquiera de las tres entidades, las manifestaciones neurológicas del sistema nervioso periférico (SNP) son frecuentes y ocurren como cualquier alteración (mononeuritis múltiple, polineuropatía, afección de pares craneales).

En general en la GPA ocurren cerca del $20 \%$ de los casos, $30-50 \%$ en la MPA y hasta en el $75 \%$ de casos con EGPA. Su presencia se considera mal pronóstico.

En cuanto al sistema nervioso central (SNC) se han descrito hemorragia, trombosis, afección central de pares craneales, infiltración meníngea y, rara vez, lesiones que ocupan espacio y alteraciones en la hipófisis. En general, este sistema se ve afectado hasta en el $10 \%$ de los enfermos en las series más grandes, con manifestaciones como cefalea, convulsiones, paresias, plejías, alteraciones conductuales, meningitis, panhipopituitarismo y diabetes insípida.

Los pares craneales raramente están involucrados en la MPA y EGPA (menos del $5 \%$ de los pacientes), pero se ven más comúnmente en la GPA (15\%). Los nervios óptico y olfatorio son afectados por la extensión de los granulomas y las porciones periféricas de los pares craneales (III-XII) pueden estar involucrados a causa de la paquimeningitis $u$ otro proceso inflamatorio. La presentación clínica de estas afecciones son la discapacidad visual, olfatoria, parálisis facial, disfagia y desórdenes sensitivos.

Las manifestaciones neurológicas en la MPA se presentan hasta en un $72 \%$ de los pacientes. La neuropatía periférica es más frecuente que la afección del sistema nervioso central y las presentaciones más frecuentes son la mononeuritis múltiple y la polineuropatía simétrica distal. Los nervios afectados más frecuentemente son: peroneo superficial, peroneo profundo, cubital, mediano y, muy raramente, los nervios craneales. En estudios de conducción nerviosa se detecta una axonopatía, mientras que en los hallazgos histológicos se demuestra una vasculitis necrosante, que se observa en biopsias de $80 \%$ de los pacientes.

Las manifestaciones del sistema nervioso central representan hasta un $30 \%$ de la afección neurológica de la MPA. Algunas de estas manifestaciones son: hemorragia cerebral, paquimeningitis e infartos cerebrales no hemorrágicos.

El compromiso neurológico en la EGPA es frecuente, siendo la neuropatía periférica mucho más prevalente que en las otras vasculitis de pequeños vasos asociadas con ANCA. La neuropatía periférica en la EGPA ocurre en un $50 \%$ a
$75 \%$ de los casos. Típicamente ocurre mononeuritis múltiple ocasionada por la lesión vasculítica de la vasa nervorum. Ocasionalmente, la lesión neuropática periférica puede tener alguna mejoría con la terapia pero en general el daño suele ser permanente y causando importante morbilidad y discapacidad funcional. Aunque el compromiso del sistema nervioso central es menos frecuente, es la segunda causa de muerte en esta entidad y se presenta con infarto o hemorragia cerebral.

En cuanto a la GPA, las manifestaciones neurológicas se producen en $22 \%$ a $54 \%$ de los pacientes. Lo más frecuente es la neuropatía periférica, especialmente la mononeuritis múltiple, mientras que la afectación del sistema nervioso central (SNC) ocurre sólo en $2 \%$ a $8 \%$ de los casos siendo las manifestaciones: la paquimeningitis hipertrófica crónica, afectación de la glándula pituitaria y la vasculitis del SNC.

En 2017, Fragoulis y colaboradores, realizaron en Grecia un estudio retrospectivo evaluando el compromiso del SNC en la GPA. Presentaron 77 pacientes de los cuales 9 (11,7\%) desarrollaron manifestaciones neurológicas. Todos los pacientes presentaron aumento de los RFA al momento de la aparición de los síntomas, y todos menos uno presentaron además compromiso de otros órganos y aumento del título de ANCA. Las manifestaciones encontradas fueron: sintomatología sensitivo-motora (33,3\%), cefalea severa y pérdida de audición (33,3\%), delirios y convulsiones (22,2\%), diplopía $(11,1 \%)$ y síntomas cerebelosos $(11,1 \%)$. Se vio que en estos pacientes el compromiso ORL y los bajos niveles de actividad de la enfermedad eran más frecuentes; así como también presentaron menor afectación a nivel pulmonar.

En un case report de 2017, la Universidad de Tsukuba, Japón, reportó un caso de un paciente masculino de 76 años, con bajo riesgo de ateroesclerosis que presentó síntomas en el hemicuerpo izquierdo incluyendo nistagmo, parálisis de las extremidades, signos piramidales, deterioro del sensorio y disestesias. Se le realizó una RMN que mostró una lesión isquémica medular medial. Basados en el hallazgo de una disfunción renal, aumento de los RFA, MPO-ANCA positivos y la ausencia de otra causa que explique la lesión (fueron descartadas diversas causas arterioescleróticas y cardiovasculares) realizaron diagnóstico de infarto medular relacionado a vasculitis ANCA. Se le instauró al paciente tratamiento con altas dosis de corticoides lo que resultó en la mejoría parcial del cuadro y rehabilitación.

La Universidad de París, Francia, en 2017 publicó una serie de reporte de casos realizado de manera retrospectiva, en el cual incluyeron 26 pacientes personales y 62 reportes de casos previos con diagnóstico de EGPA. Encontraron que un $56 \%$ de los pacientes presentaron compromiso del SNP mientras que un $86 \%$ tuvieron manifestaciones a nivel del SNC. De este último $86 \%$, el $2 \%$ presentó el cuadro neurológico antes del diagnóstico de EGPA y un 12\% lo manifestó en los primeros 24 meses de seguimiento. La mayoría desarrolló lesiones isquémicas a nivel cerebral (52\%), seguidos de hemorragia cerebral o subaracnoidea (24\%), disminución de la agudeza visual en 28 pacientes (33\%), de los cuales 15 fueron por neuritis óptica, 9 por oclusión de la arteria central de la retina, y 4 por ceguera cortical. 18 pacientes presentaron compromiso de los pares craneales. En 25 de la totalidad de 
los pacientes se presentaron más de uno de estos síntomas.

En cuanto al tratamiento, $43 \%$ de los pacientes tuvo adecuada respuesta sin secuelas neurológicas, mientras que otro $43 \%$ continuó con secuelas y $14 \%$ fue refractario al tratamiento. Luego de un seguimiento de 36 meses, 11 pacientes murieron, 5 de ellos por hemorragia cerebral.

En el año 2015, la revista Rheumatology publicó un estudio retrospectivo sobre una cohorte alemana de 144 pacientes con diagnóstico de MPA. Observaron que el 56\% de los pacientes presentó compromiso del SNP al inicio de la enfermedad (tanto en forma de mononeuritis múltiple, como neuropatía sensorial pura) y en total el 61\% presentó manifestaciones en el total del seguimiento. En cuanto a compromiso de SNC, este se vio afectado solo en un $3 \%$ de los pacientes al inicio de la enfermedad y en un $5 \%$ en el total del follow-up. Se concluyó que la mortalidad de estos pacientes se encuentra aumentada en el caso de que haya compromiso neurológico.

Camarra-Lenarroy y colaboradores presentaron, en el año 2015, el reporte de un caso de una paciente femenina de 38 años con antecedentes de asma que comenzó con parestesias de miembros inferiores la cual fue progresando a una parálisis simétrica ascendente. Se le realizaron pruebas de conducción nerviosa, las cuales no fueron suficientes para descartar un síndrome de Guillain-Barré. El laboratorio mostraba una marcada eosinofilia (mayor al 50\%), presentaba lesiones purpúricas en miembros inferiores y la RMN reveló pansinusitis, por lo cual se realizó el diagnóstico de EGPA. La paciente mejoró con la administración de esteroides. Posteriormente el resultado de ANCA fue positivo.

En el año 2011, la revista Rheumatology (Oxford) publicó un research report sobre el compromiso del SNP en las vasculitis asociadas a ANCA. Los datos fueron extraídos de trials conducidos por el grupo EUVAS.

En total analizaron 506 pacientes (237 con MPA, 269 con GPA). La presencia de neuropatía motora o sensitivo-motora al inicio de ambas enfermedades fue la misma: 7\%. En total, 48 pacientes desarrollaron compromiso neurológico en algún momento de la enfermedad. La parálisis de pares craneales fue más común en la GPA (17\% de los pacientes) que en la MPA (8\%).

En un estudio realizado en el Hospital Durand, en 2011 se vio que la frecuencia de compromiso neurológico en las vasculitis asociadas a ANCA fue menor que en la literatura; de 47 pacientes, 7 (15\%) presentaron compromiso del SNP y 3 (6\%) del SNC, observándose principalmente el compromiso neurológico en la MPA.

En 2007, el Complejo Hospitalario Universitario de Santiago de Compostela, España, presentó un caso clínico de un paciente masculino de 47 años que consultó por manifestaciones ORL y se objetivó paresia del III y VI pares craneales izquierdos y amaurosis del ojo izquierdo. Se realizó una (RNM) cerebral que demostró una lesión expansiva submucosa de $3 \mathrm{~cm}$ de diámetro en el cauum de Meckel izquierdo, con realce tras la administración de contraste, que obstruía la trompa de Eustaquio y se extendía a la base del cráneo. Se realizó una biopsia, la cual fue informada como inflamación crónica granulomatosa, necrotizante, con células gigantes multinucleadas tipo Langhans, con tinción Ziehl-Neelsen negativa. Se realizaron dos biopsias más que obtuvieron tejido inflamatorio agudo y crónico por lo cual inició tratamiento con corticoides. Al realizar tratamiento con corticoides se resolvió la paresia de los pares craneales y mejoró su estado general. En ese momento en la exploración física se destacaba: pupila en midriasis media, poco reactiva, y amaurosis en el ojo izquierdo, ligera paresia de la extremidad inferior izquierda, reflejo cutáneo plantar derecho indiferente e izquierdo flexor y lesiones de apariencia purpúrica en ambas extremidades inferiores. En el laboratorio se destacaba una anemia normocítica normocrómica, leucocitosis con neutrofilia y VSG $107 \mathrm{~mm}$. La orina presentaba indicios de proteínas, 20-30 leucocitos y abundantes hematíes y la orina de $24 \mathrm{~h}$ una proteinuria de $0,07 \mathrm{~g} / \mathrm{l}$. Los ANCA fueron positivos a título 1/80 con anticuerpos antiproteinasa 3 positivos. La tomografía axial computarizada (TAC) toracoabdominal mostró tres nódulos pulmonares cavitados, pequeñas lesiones focales hepáticas y esplénicas. La electromiografía de las extremidades inferiores puso de manifiesto la existencia de una polineuropatía motora axonal. Se instauró tratamiento oral con prednisona y ciclofosfamida, con mejoría del cuadro clínico y del laboratorio.

En un case report del año 2006, hecho en Baltimore, se describen 6 pacientes con compromiso del SNC, 2 con paquimeningitis, 3 con compromiso de la glándula pituitaria y 1 con vasculitis cerebral. De estos 6 pacientes, 2 presentaron el compromiso neurológico al inicio de la enfermedad mientras que 4 lo presentaron entre 5-18 años después del diagnóstico.

\section{Diagnóstico}

El primer paso ante un paciente con sospecha de vasculitis es obtener un diagnóstico de certeza, preferiblemente con confirmación histológica, de la presencia de vasculitis, ya que su diagnóstico diferencial es extenso. En un segundo término se intentará concretar el diagnóstico definitivo del subtipo de vasculitis. Sin embargo, esta distinción no suele ser tan trascendental, puesto que el tratamiento en general se fundamenta en una inmunosupresión más o menos intensa que dependerá más del grado de afectación sistémica.

El diagnóstico precoz requiere un alto grado de sospecha ante un paciente con síntomas sugestivos. La presencia de fiebre de origen desconocido, afección multisistémica, mononeuritis múltiple, púrpura o lesiones cutáneas, insuficiencia renal, infiltrados pulmonares y hematuria, afección otorrinolaringológica destructiva, o la aparición de manifestaciones isquémicas en cualquier localización, especialmente si son múltiples $u$ ocurren en personas jóvenes o sin factores de riesgo vascular, son escenarios clínicos que nos deben alertar sobre la posible existencia de una vasculitis ANCA.

El diagnóstico del subtipo de vasculitis se sustenta en la conjunción de datos clínicos e histológicos con el soporte de las exploraciones complementarias, especialmente la determinación de los ANCA. Como ya se ha comentado, siempre que sea posible es deseable confirmar el diagnóstico mediante biopsia de cualquier tejido afectado, puesto que 
la única prueba definitiva de la existencia de una vasculitis es su evidencia histológica. La utilidad clínica de unos ANCA positivos en el diagnóstico inicial de una vasculitis es indiscutible pero limitada y siempre muy influenciada por el cuadro clínico del paciente. Además, también se ha de tener en cuenta que los ANCA son negativos en un $40 \%$ de los pacientes con GPA locorregional y en un $10 \%$ de los casos con enfermedad generalizada, en un $30 \%$ de los pacientes con MPA y en cerca de un 50\% de los casos de EGPA. Por lo tanto, la negatividad de los ANCA no permite excluir el diagnóstico. Además, el estatus de los ANCA puede cambiar a lo largo del tiempo y en un paciente con GW en fase inicial locorregional pueden ser negativos y positivizarse cuando la enfermedad se generaliza.

En un paciente con un cuadro clínico compatible y con compromiso vital, si han sido adecuadamente descartadas otras posibilidades, la presencia de unos ANCA positivos con patrón anti-PR3 o anti-MPO son un argumento casi definitivo a favor de la presencia de una Vasculitis asociada a ANCA, lo que nos ayuda en la decisión de iniciar un tratamiento inmunosupresor sin demoras, antes de realizar una biopsia o antes de obtener el resultado, si es que esta ya se ha hecho.

Además, otros exámenes complementarios son de utilidad. En el laboratorio puede verse anemia normocítica, normocrómica, aumento de los RFA, leucocitosis, eosinofilia marcada en el caso de la EGPA.

El examen de orina será útil para evaluar el compromiso renal en estas entidades, la presencia de cilindros, hematíes y proteinuria en una muestra de orina de 24 h, más los hallazgos en la función renal sérica nos hará sospechar la probable afectación renal.

La TAC de tórax y senos paranasales resulta de utilidad para evaluar el compromiso de la vía aérea superior e inferior, el cual se manifiesta en forma de infiltrados bilaterales, nódulos y cavitaciones.

En cuanto al compromiso del sistema nervioso, los estudios de conducción nerviosa pueden resultar útiles para verificar la presencia y la extensión de la neuropatía.

La biopsia de nervio, usualmente del nervio sural establece definitivamente la presencia de vasculitis. En cuanto al SNC, tanto la TAC como la RMN son de utilidad para definir infartos, hemorragias, lesiones ocupantes de espacio, alteraciones de la sustancia blanca y de la médula espinal. Demostrar la presencia de vasculitis puede ser difícil ya que los vasos afectados son los de pequeño calibre y la angiografía puede no mostrar alteraciones. La punción lumbar puede llegar a ser necesaria en el caso de sospecha de hemorragia subaracnoidea o para descartar la presencia de infección meníngea.

\section{Tratamiento}

En cuanto al tratamiento, debemos tener en cuenta que el compromiso neurológico es parte de una enfermedad sistémica generalizada, por lo cual el tratamiento está dirigido hacia la totalidad de la entidad. No hay tratamiento específico para el compromiso neurológico en sí.

La clave del éxito es iniciar el tratamiento lo más tempranamente posible; está basado en la utilización de agentes inmunosupresores y consta de dos fases: la inducción, la cual tiene un período entre 3 a 6 meses y el mantenimiento el cual debe prolongarse por 18 a 24 meses.

La terapia estándar se inicia con la inducción con altas dosis de corticoides (prednisona $1 \mathrm{mg} / \mathrm{kg}$ ) por aproximadamente un mes y con posterior tappering del mismo. A este fármaco se adicionará además otro inmunosupresor como, por ejemplo, ciclofosfamida ( $1 \mathrm{~g} / \mathrm{mes})$.

La terapia de mantenimiento está basada en bajas dosis de esteroides, azatioprina o metotrexato.

Los casos severos pueden requerir pulsos de corticoides intravenosos (metilprednisolona 500-1000 mg/día por 3-5 días).

La plasmaféresis puede resultar beneficiosa, pero la evidencia es limitada en el caso del compromiso neurológico.

En el caso de baja actividad de la enfermedad, hay estudios que avalan el uso de bajas dosis de corticoides, metotrexato o micofenolato. La leflunomida, que también se ha usado en casos de baja actividad, debe evitarse en el caso de que haya manifestaciones del sistema nervioso ya que tiene efectos adversos neurotóxicos.

Estudios recientes probaron la eficacia de rituximab tanto para inducción como para mantenimiento y, en acuerdo con las guías británicas, se puede usar indistintamente con la ciclofosfamida, si bien aún no se encuentra instaurado como tratamiento de primera línea. No existen suficientes datos de la eficacia del rituximab en cuanto a la mejoría de los síntomas neurológicos, sin embargo algunos reportes de casos se muestran promisorios.

Otros anticuerpos monoclonales (infliximab, etanercept) tienen baja efectividad, y algunos (belimumab, mepolizumab) se encuentran siendo investigados.

Hay también algunos reportes alentadores sobre el uso de gammaglobulina intravenosa, sobre todo para pacientes con manifestaciones neurológicas residuales.

\section{Conclusión}

Las vasculitis asociadas a ANCA es un grupo heterogéneo de enfermedades que causan daño e inflamación en vasos de pequeño y mediano calibre; pueden ocasionar afectación en numerosos órganos y sistemas, entre los mismos se encuentra el sistema nervioso, tanto central como periférico.

La afectación neurológica de estas entidades puede presentarse con un sinfín de síntomas, por lo cual es de suma importancia tenerlos en cuenta y sospecharlos para poder llegar a un diagnóstico y tratamiento adecuado y precoz que son factores decisivos para lograr una buena respuesta terapéutica, alcanzar la remisión, prevenir el daño crónico y dar una mejor calidad de vida a los pacientes que conviven con la enfermedad.

Así mismo no hay que olvidar que el tratamiento de la afectación neurológica en estas enfermedades es de manejo multidisciplinario y que es esencial la cooperación entre las distintas especialidades (reumatología, neurología, rehabilitación, etc.) para poder darle al paciente un cuidado y un tratamiento que abarque todos los aspectos de la enfermedad. 


\section{BIBLIOGRAFÍA}

1. Kelley's text book of Rheumatology, 9na edición. Editorial Elsevier

2. Manual SER de diagnóstico y tratamiento de las enfermedades reumáticas autoinmunes sistémicas. 1ra edición. Sociedad española de Reumatología. Editorial Elsevier.

3. www.vasculitis.org

4. www.rheumatology.org

5. Fragoulis GE, Lionakis S. Central nervous system involvement in patients with GPA: a single center retrospective study. Clin. Rheumatol. 2017. DOI: 10.1007/s10067-017-3835-y

6. Kumi Yanagiha, Kazuhiro Ishii. Medial medullary infarction caused by antineutrophil cytoplasmic antibody related vasculitis. Medicine (Baltimore) 2017, Aug; 96(33):e7722. DOI: 10.1097/MD0000000000007722

7. André R, Cottin V, Saraux JL. Central nervous system involvement in eosinophilic granulomatosis with polyangiitis (Churg-Strauss): report of 26 patients and review of the literature. Autoimmun Rev 2017 Sep; 16(9):963969. DOI: 10.1016/j.autrev.2017.07.007

8. Wludarczyk A, Szczeklik W. Neurological manifestation in ANCA associated vasculitis. Assessment and treatment. Expert rev Neurother 2016 Aug; 16(8):861-3. DOI: 10.1586/14737175.2016.1165095

9. Suppiah R, Hadden RD. Peripheral neuropathy in ANCAassociated vasculitis outcomes from the european vasculitis study group trials. Rheumatology (Oxford) 2011 Dec; 50(12):2214-22. DOI: 10.1093/rheumatology/ker266

10. Schirmer JH, Wright MN. Clinical presentation and longterm outcome of 144 patients with microscopic polyangiitis in a monocentric German cohort. Rheumatology (Oxford). 2016 Jan; 55(1):71-9. DOI: 10.1093/rheumatology/kev286

11. Camara-Lemarroy $C R$, Infante-Valenzuela A. Eosinophilic Granulomatosis with Polyangiitis Presenting as Acute Polyneuropathy Mimicking Guillain-Barre Syndrome. Case Rep Neurol Med. 2015; 2015:981439. DOI: 10.1155/2015/981439 12. Mauricio Restrepo, Luis Alonso González, Gloria Vásquez.
Síndrome de Churg-Strauss: a propósito de un caso con manifestaciones poco usuales. Revista colombiana de reumatología Marzo 2009; 16(1):112-119.

13. Carolina Muñoz Grajales, Álvaro Arbeláez Cortés, Luis Fernando Arias Restrepo. Poliangítis microscópica: reporte de un caso con enfermedad renal, pulmonar y neurológica, y revisión de la literatura. Revista colombiana de reumatología, Junio 2011, 18(2):121-134.

14. Carlos Francisco Pérez-Beltrán, María julia Garza Zúñiga. Vasculitis y sus manifestaciones neurológicas. Med Int Méx 2015; 31:324-336.

15. Raimundo López-Rodríguez, Jorge García-González. Manifestaciones neurológicas de la granulomatosis de Wegener. Caso clínico. Rev Méd Chile 2007; 135:913-916.

16. María Virginia Paolini, Juan Pablo Ruffino. Vasculitis asociadas a anticuerpos anti-citoplasma de neutrófilos. Clínica y tratamiento. Medicina (B. Aires) vol.73 no.2 Ciudad Autónoma de Buenos Aires abr. 2013.

17. Seror R, Mahr A. Central nervous system involvement in Wegener granulomatosis. Medicine (Baltimore). 2006 Jan; 85(1):54-65.

18. Bielsa I. Actualización en la nomenclatura de las vasculitis. Conferencia de Consenso Internacional de Chapel Hill, 2012. Actas Dermosifiliogr. 2015; 106(8):605-608.

19. Saech J, Owczarczyk K, Rosgen S, et al. Successful use of rituximab in a patient with Churg-Strauss syndrome and refractory central nervous system involvement. Ann Rheum Dis. 2010; 69 (6):1254-1255.

20. Sharma A, Kumar S, Wanchu A, et al. Successful treatment of hypertrophic pachymeningitis in refractory Wegener's granulomatosis with rituximab. Clin Rheumatol. 2010; 29(1):107-110.

21. De Joode AA, Sanders JS, Smid WM, et al. Plasmapheresis rescue therapy in progressive systemic ANCA-associated vasculitis: single-center results of stepwise escalation of immunosuppression. J Clin Apher. 2014; 29(5):266-272.

22. Martin K, Bentaberry F, Dumoulin C, et al. Peripheral neuropathy associated with leflunomide: is there a risk patient profile? Pharmacoepidemiol Drug Saf. 2007; 16(1):74-78. 